\title{
Topological Representation of Dual Pairs of Oriented Matroids
}

\author{
Thomas H. Brylawski ${ }^{1}$ and Günter M. Ziegler ${ }^{2}$ \\ ${ }^{i}$ Department of Mathematics, University of North Carolina, \\ Chapel Hill, NC 27514, USA \\ ${ }^{2}$ Konrad-Zuse-Zentrum für Informationstechnik Berlin, \\ Heilbronner Strasse 10, 1000 Berlin 31, Federal Republic of Germany \\ ziegler@zib-berlin.de
}

\begin{abstract}
We prove that every pair of dual oriented matroids can be represented in complementary subspheres of an arrangement of pseudospheres.
\end{abstract}

Among the many ways to view oriented matroids as geometrical objects, we consider two that have special properties:

- Bland's analysis of complementary subspaces in $\mathbb{R}^{n}[2]$ has the special feature that it simultaneously and symmetrically represents a realizable oriented matroid and its dual.

- Lawrence's topological representation of oriented matroids by arrangements of pseudospheres [4] has the advantage of yielding a faithful picture also in the general case of nonrealizable oriented matroids.

In this note we prove a "Topological Representation Theorem for Dual Pairs," which combines these two points of view.

We refer to Chapter 1 of [1] for an exposition of the theory of oriented matroids. Here we only review some notation and fix terminology.

Bland's [2, Section 3], [1, Section 1.2(d)] set-up is as follows. Let $\xi_{B}$ be a subspace of $\mathbb{R}^{n}$ of dimension $r$. The intersections of the coordinate hyperplanes $H_{i}=\left\{\mathrm{x} \in \mathbb{R}^{n}: x_{i}=0\right\}$ with $\xi$ determine an arrangement of hyperplanes $\left\{\xi \cap H_{i}: 1 \leq i \leq n\right\}$ in $\xi$, and with it a (realizable) oriented matroid $\mathscr{M}$ of rank $r$ on $\{1, \ldots, n\}$. In the same way, the orthogonal complement $\xi^{\perp}$ of dimension $n-r$ determines an arrangement in $\xi^{\perp}$ that represents $\mathscr{M}^{*}$. 
Now write $\xi$ and $\xi^{\perp}$ as intersections $\xi^{\perp}=\bigcap_{j=n+1}^{n+r} H_{j}^{\prime}$ and $\xi=\bigcap_{j=n+r+1}^{2 n} H_{j}^{\prime}$ of hyperplanes $H_{j}^{\prime} \subseteq \mathbb{R}^{n}$. This construction encodes the realizable oriented matroid $\mathscr{M}$ and its dual $\mathscr{M}^{*}$ into an arrangement of $2 n$ hyperplanes $H_{i}, H_{j}^{\prime}$ in $\mathbb{R}^{n}$ for $1 \leq i \leq n$ and $n+1 \leq j \leq 2 n$. In view of this, the Topological Representation Theorem of Lawrence suggests a generalization that encodes a general pair of dual oriented matroids into an arrangement of $2 n$ pseudospheres in $S^{n-1}$, stated below as Theorem 1.

For this, recall that a pseudosphere is the image of a coordinate sphere $S_{i}=\left\{\mathrm{x} \in S^{n-1}: x_{i}=0\right\}$ (for $1 \leq i \leq n$ ) under a homeomorphism $h: S^{n-1} \rightarrow S^{n-1}$. The complement of a pseudosphere $S$ in $S^{n-1}$ has two components $S^{+}$and $S^{-}$, called the sides of $S$.

A pseudosphere arrangement (or pseudoarrangement) is a family $\mathscr{A}=\left(S_{e}\right)_{e \in E}$ of pseudospheres in $S^{n-1}$ such that, for $A \subseteq E$, the intersection $S_{A}=\bigcap_{e \in E} S_{e}$ is a sphere of some dimension, $S_{E}=\varnothing$, and, for $\varnothing \neq S_{A} \nsubseteq S_{e}$, the intersection $S_{A} \cap S_{e}$ is a pseudosphere in $S_{A}$ with sides $S_{A} \cap S_{e}^{+}$and $S_{A} \cap S_{e}^{-}$. A pseudoarrangement is signed if, for every $S_{e} \in \mathscr{A}$, a positive side $S_{e}^{+}$is chosen.

The intersections $S_{A}$ are called subspheres of $\mathscr{A}$. Two subspheres $S_{A}$ and $S_{B}$ are complementary if, for some $r, S_{A}$ is an $(r-1)$-sphere and $S_{B}$ is an $(n-r-1)$-sphere, with $S_{A} \cap S_{B}=\varnothing$.

The Topological Representation Theorem [4, Chapter IV], [1, Section 1.4 and Chapter 5] states that there is a bijection between oriented matroids of rank $r$ on $n$ elements and equivalence classes of signed arrangements of $n$ pseudospheres in $S^{r-1}$. Under this bijection, the $k$-subspheres of an arrangement of pseudospheres correspond to contractions of the oriented matroid of rank $k+1$; in particular, the cocircuits of the oriented matroid can be identified with the vertices of the pseudoarrangement.

Theorem 1 (Topological Representation of Dual Pairs). Let $\mathscr{M}$ be an oriented matroid of rank $r$ on $\{1, \ldots, n\}$. There is a signed arrangement of $2 n$ pseudospheres $\mathscr{A}=\left(S_{i}\right)_{1 \leq i \leq 2 n}$ in $S^{n-1}$ so that:

- $S_{i}=\left\{\mathbf{x} \in S^{n-1}: x_{i}=0\right\}$ for $1 \leq i \leq n$ (that is, $\mathscr{A}$ contains the "frame" of linear coordinate spheres).

- The $(r-1)$-subsphere $S_{B}:=S_{n+r+1} \cap \cdots \cap S_{2 n}$ and the $(n-r-1)$-subsphere $S_{A}:=S_{n+1} \cap \cdots \cap S_{n+r}$ form a pair of complementary subspheres in $S^{n-1}$.

- The arrangement $\left(S_{i} \cap S_{B}\right)_{1 \leq i \leq n}$ is a topological representation of $\mathscr{M}$ in $S_{B}$.

- The arrangement $\left(S_{i} \cap S_{A}\right)_{1 \leq i \leq n}$ is a topological representation of $\mathscr{M}^{*}$ in $S_{A}$.

In view of the Topological Representation Theorem, Theorem 1 can be reduced to the following construction of an oriented matroid that has $\mathscr{M}$ and $\mathscr{M}^{*}$ as complementary minors.

Theorem 2 (Representation of Dual Pairs as Complementary Minors). For every oriented matroid $\mathscr{M}$ of rank $r$ on the ground set $E=\{1, \ldots, n\}$, there exists an oriented matroid $\hat{\mathscr{M}}$ of rank $n$ on the ground set $\hat{E}=\{1, \ldots, 2 n\}=E \cup A \cup B$ with 
$A:=\{n+1, \ldots, n+r\}$ and $B:=\{n+r+1, \ldots, 2 n\}$, such that

$$
\begin{aligned}
& \hat{\mathscr{M}} \backslash A / B=\mathscr{M}, \\
& \hat{\mathscr{M}} / A \backslash B=\mathscr{M}^{*} .
\end{aligned}
$$

Proof. For the following, we relabel the ground set such that $\{1, \ldots, r\}$ is a basis of $\mathscr{M}$. Now let $\mathscr{M}_{1}$ be the oriented matroid on $\hat{E}$ that is obtained by extending $\mathscr{M}$ by elements $n+i$ that are parallel to the elements $i$ for $1 \leq i \leq r$, and that are loops for $r+1 \leq i \leq n$. Similarly, let $\mathscr{M}_{2}$ be the oriented matroid on $\hat{E}$ that is obtained by extending $\mathscr{M}^{*}$ by elements $n+i$ that are loops for $1 \leq i \leq r$ and that are parallel to the elements $i$ for $r+1 \leq i \leq n$.

$\mathscr{M}_{1}$ and $\mathscr{M}_{2}$ are matroids of ranks $r$ and $n-r$ on $\hat{E}$ that have disjoint bases. Thus their union $\hat{\mathscr{M}}:=\mathscr{M}_{1} \cup \mathscr{M}_{2}$ (see [5] and Section 7.6 of [1]) is an oriented matroid of rank $n$ on $\hat{E}$. We claim that $\hat{\mathscr{M}}$ has the required properties. To see this, we use an explicit description of oriented matroid union by Lawrence and Weinberg [5], [1, Proposition 7.6.4]: if $A_{1}$ and $B_{1}$ are disjoint (ordered) bases of $\mathscr{M}_{1}$ and of $\mathscr{M}_{2}$, so that $\left(A_{1}, B_{1}\right)$ is the lexicographically smallest permutation of $A_{1} \cup B_{1}$ for which the first $r_{1}$ elements form a basis of $\mathscr{M}_{1}$ and the other $r_{2}$ elements form a basis of $\mathscr{M}_{2}$, then

$$
\chi_{\mathscr{M}_{1} \cup \mathscr{M}_{2}}\left(A_{1} \cup B_{1}\right)=\chi_{\mathscr{M}_{1}}\left(A_{1}\right) \cdot \chi_{\mathscr{M}_{2}}\left(B_{1}\right) .
$$

In our situation, let $A_{1}$ be an $r$-subset of $\{1, \ldots, n\}$. If $A_{1}$ is not a basis of $\mathscr{M}_{1}$, then $A_{1} \cup B$ is not a basis of $\mathscr{M}_{1} \cup \mathscr{M}_{2}$, since the elements of $B$ are loops in $\mathscr{M}_{1}$, and thus $\chi_{\left(\mathscr{M}, \cup \mathscr{M}_{2}\right) A / B}\left(A_{1}\right)=\chi_{\mathscr{M}}\left(A_{1}\right)=0$. If $A_{1}$ is a basis of $\mathscr{M}_{1}$, then $A_{1} \cup B$ is a basis of $\mathscr{M}_{1} \cup \mathscr{M}_{2}$, and the Lawrence-Weinberg formula yields

$$
\chi_{\left(\mathscr{M}, \cup \mathscr{M}_{2} \backslash A / B\right.}\left(A_{1}\right)=\chi_{\left(\mathscr{M}_{1} \cup \mathscr{M}_{2}\right)}\left(A_{1} \cup B\right)=\chi_{\mathscr{M}_{1}}\left(A_{1}\right) \cdot \chi_{\mathscr{M}_{2}}(B)=\chi_{\mathscr{M}}\left(A_{1}\right),
$$

which proves $\left(\mathscr{M}_{1} \cup \mathscr{M}_{2}\right) \backslash A / B=\mathscr{M}$. Analogously, we get $\left(\mathscr{M}_{1} \cup \mathscr{M}_{2}\right) \backslash B / A=\mathscr{M}^{*}$.

Theorem 2 has a straightforward analogue for ordinary matroids. The main difference is that in the unoriented case the construction of a union is unique, while the oriented construction involves a lot of choice. However, even in the unoriented case the conditions of Theorem 2 do not uniquely determine $\hat{M}$.

In the case where $\mathscr{M}$ is realizable, the oriented matroid $\hat{\mathscr{M}}$ constructed from it is again realizable. Namely, if $\mathscr{M}$ can be represented by $(I \mid C)$, where $I$ denotes an identity matrix, then $\mathscr{M}_{1}$ is represented by $(I|C| I \mid 0)$ and $\mathscr{M}_{2}$ is represented by $\left(-C^{t}|I| 0 \mid I\right)$. Now let $\left(-C^{t}|I| 0 \mid I\right)^{\varepsilon}$ be the matrix obtained by multiplying the $i$ th column by $\varepsilon^{2 n-i}$ for all $i \in\{1, \ldots, 2 n\}$ and $\varepsilon>0$ sufficiently small. Then the combined matrix

$$
\left.\begin{array}{cccc}
I & C & I & 0
\end{array}\right)
$$


is a representation of $\hat{\mathscr{M}}$, see [5] and Proposition 8.2.7 of [1]. A similar statement holds for ordinary matroids when represented over a sufficiently large field, see Proposition 7.6.1 of [3].

The construction of Theorem 2 seems to be new. We expect that it should have other applications, facilitating the use and the interpretation of (oriented) matroid duality, to the analysis of linear programming algorithms on oriented matroids, etc.

\section{References}

1. A. Björner, M. Las Vergnas, B. Sturmfels, N. White, and G. M. Ziegler: Oriented Matroids, Cambridge University Press, Cambridge, 1993.

2. R. G. Bland: A combinatorial abstraction of linear programming, J. Combin. Theory Ser. B 23 (1977), 33-57.

3. T. Brylawski: Constructions, in: Theory of Matroids (N. White, ed.), Cambridge University Press, Cambridge, 1986, pp. 127-223.

4. J. Folkman and J. Lawrence: Oriented matroids, J. Combin. Theory Ser. B 25 (1978), 199-236.

5. J. Lawrence and L. Weinberg: Unions of oriented matroids, Linear Algebra Appl. 41 (1981), 183-200.

Received December 23, 1991, and in revised form February 18, 1993. 